\title{
Ectopic pregnancy in a case of congenital mullerian anomaly: a diagnostic dilemma
}

\author{
Sanjay Brahmbhatt ${ }^{1}$, Amrita Makhija ${ }^{2}$, Jayna Brahmbhatt ${ }^{1}$, Yagnesh V. Patel ${ }^{1 *}$
}

\author{
${ }^{1}$ Department of Gynecological Endoscopy, Himalaya Hospital, Anand, Gujarat, India \\ ${ }^{2}$ Department of Obstetrics and Gynecology, Himalayan Institute of Medical Sciences, Dehradun, Uttarakhand, India
}

Received: 22 May 2020

Accepted: 30 June 2020

\section{*Correspondence:}

Dr. Yagnesh V. Patel,

E-mail: yvpatel1989@gmail.com

Copyright: () the author(s), publisher and licensee Medip Academy. This is an open-access article distributed under the terms of the Creative Commons Attribution Non-Commercial License, which permits unrestricted non-commercial use, distribution, and reproduction in any medium, provided the original work is properly cited.

\begin{abstract}
Ectopic or extrauterine pregnancy occurring in a case with mullerian defect is very rare and poses diagnostic challenges. Undescended and non-communicating fallopian tubes are extremely rare mullerian anomalies. Here authors present a case of ectopic pregnancy occurring in an undescended non-communicating fallopian tube in a patient with unicornuate uterus with absent horn, which was managed laparoscopically. A 32-year-old lady, diagnosed case of left unicornuate uterus with absent right horn, was referred to us with the suspicion of ruptured ectopic pregnancy. The abdominopelvic ultrasound showed a soft tissue lesion of size $32 \times 24 \mathrm{~mm}$, towards the right lateral pelvic wall near the iliac vessels, with increased vascularity on colour flow doppler. The patient underwent laparoscopy which showed left sided unicornuate uterus with normal left tube and ovary. The right uterine horn was absent. An undescended right ovary and tube were found attached to the peritoneum at the level of pelvic brim along the right lateral pelvic wall. Right sided tubal ectopic pregnancy with rupture was present along with $300-350 \mathrm{cc}$ of hemoperitoneum. The patient was treated with laparoscopic right sided total salpingectomy. In patients with unicornuate uterus and atypical presentation, ectopic pregnancy should be ruled out in an undescended noncommunicating fallopian tube. Salpingectomy of incidentally diagnosed non-communicating fallopian tubes is recommended to prevent future ectopic pregnancy.
\end{abstract}

Keywords: Ectopic pregnancy, Laparoscopy, Mullerian anomaly, Unicornuate uterus, Undescended fallopian tube

\section{INTRODUCTION}

Ectopic or extrauterine pregnancy accounts for $1.3-2.4 \%$ of all pregnancies. ${ }^{1}$ With better understanding and the advancement in the diagnostic modalities, almost all of the ectopic pregnancies are being detected and managed timely. However, ectopic pregnancies occurring in cases with mullerian defects still pose diagnostic challenges.

Here, authors present a case of ectopic pregnancy occurring in an undescended non-communicating fallopian tube in a patient with unicornuate uterus with absent horn.

\section{CASE REPORT}

A 32-year-old lady was referred to us from a private doctor with a suspicion of ruptured ectopic pregnancy. She complained of on and off pain in the lower abdomen for the past 2 weeks. The pain was mild to moderate in intensity. She also had history of a syncopal attack 1 week ago with an associated episode of increase in the abdominal pain. Her last menstrual period was 8 days ago and her previous menstrual cycles were regular, although she noticed decreased flow in the last three cycles. The patient was parity two, with two previous uneventful cesarean deliveries, 9 years and 5 years ago. She had also undergone diagnostic laparoscopy 10 years ago for the 
evaluation of infertility, when she was diagnosed to be a case of left unicornuate uterus with absent right horn (Buttram and Gibbons' classification class IIB or European Society of Human Reproduction and Embryology-European Society for Gynecological Endoscopy (ESHRE/ESGE) classification class U4b). She was also found to have absence of right kidney.

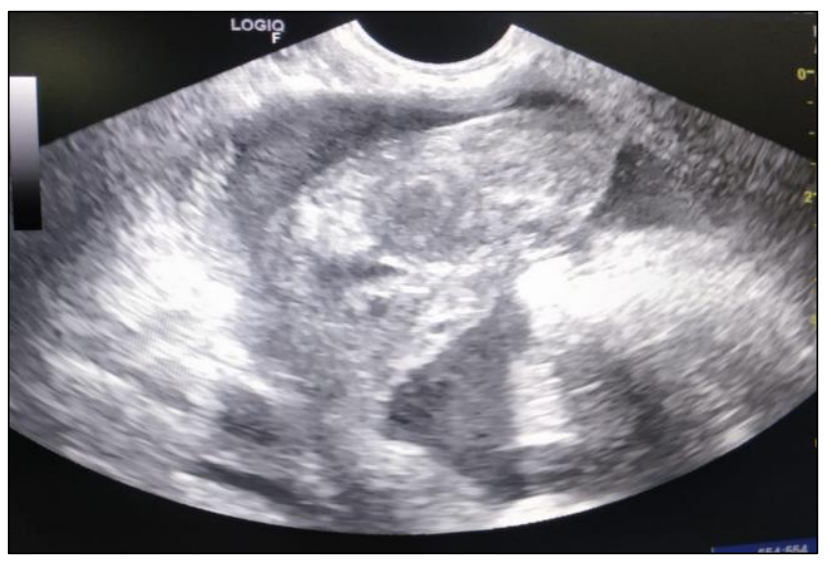

Figure 1: Ultrasound of ectopic pregnancy towards the right lateral pelvic wall.

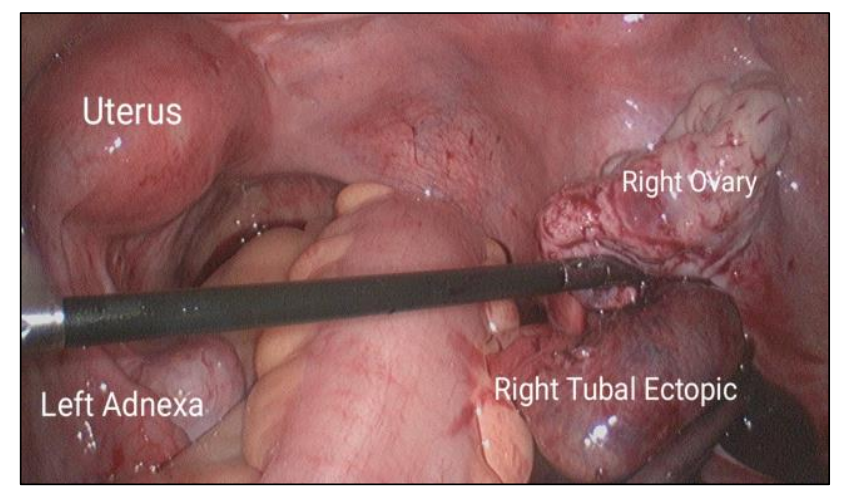

Figure 2: Laparoscopic of ectopic pregnancy in undescended and non-communicating right fallopian tube.

The patient was conscious and well oriented. She had mild pallor on examination. Her pulse rate was 96 beats per minute and blood pressure was $100 / 70 \mathrm{mmHg}$. Rest of the general physical examination and systemic examination was unremarkable. On abdominal examination, there was mild tenderness on deep palpation in the lower abdomen. Per speculum examination was unremarkable. On per vaginal examination fullness was present in the pouch of douglas. However, cervical motion tenderness could not be elicited.

The patient's haemoglobin was $10.2 \mathrm{mg} / \mathrm{dl}$. Other blood investigations were within normal limits. Her urinary human chorionic gonadotropin (HCG) test was positive. The abdominal and pelvic ultrasound was repeated at the study hospital which showed bilaterally normal adnexa and no evidence of intrauterine pregnancy. However, a soft tissue lesion of size $32 \times 24 \mathrm{~mm}$ was seen towards the right lateral pelvic wall near the iliac vessels (Figure 1). The lesion showed increased vascularity with colour flow doppler. The pouch of Douglas contained mild free fluid with internal echoes. The absence of right kidney was also noted.

An abdominal ectopic pregnancy was suspected based on the above evaluation and the patient was planned for laparoscopy. On laparoscopic evaluation by standard equipment, presence of around 300-350 cc of hemoperitoneum was noted. Left sided unicornuate uterus with normal left tube and ovary was present. The right uterine horn was absent. An undescended right ovary and tube were found attached to the peritoneum along the right lateral pelvic wall at the level of the pelvic brim. Right sided tubal ectopic pregnancy with rupture was present (Figure 2). The patient underwent laparoscopic right sided total salpingectomy and specimen was sent for histopathological evaluation. The post-operative period was uneventful and the patient was discharged after 2 days. The histopathological examination report confirmed the presence of tubal ectopic pregnancy.

\section{DISCUSSION}

The female internal genital organs develop from the fusion of the two paired mullerian or paramesonephric ducts. Anomalies in the development of mullerian ducts are seen in $4-7 \%$ of women with normal fertility. ${ }^{2}$ Unicornuate uterus is seen when a single mullerian duct develops and agenesis or abnormal development of the other duct occurs. Almost a third of the cases with unicornuate uterus, have an absent opposite horn, although as authors found in this case, fallopian tubes may be present on both the sides. Undescended and noncommunicating fallopian tubes are extremely rare mullerian anomalies and little about these has been described in literature. Isolated ovarian maldescent may also occur rarely but is seen in up to $40 \%$ of the cases of unicornuate uterus. ${ }^{3}$

Tubal ectopic pregnancy in an undescended fallopian tube has been reported in literature. ${ }^{4,5}$ The first case was reported in $1987 .^{6}$

The condition has posed diagnostic challenges and commonly the diagnosis has been missed prior to rupture, leading to increase in morbidity. Atypical symptoms such as chest pain or shortness of breath have been noted in a case where the fallopian tube was found lateral to spleen. ${ }^{7}$ Tubal ectopic pregnancy has also been noted in an undescended non-communicating tube under the hepatic flexure of colon, causing right upper abdominal pain. ${ }^{8}$ The mechanism for pregnancy in non-communicating fallopian tubes is considered to be transperitoneal migration of gametes/embryo. Therefore, some authors suggest the removal of non-communicating tubes to decrease the risk of future ectopic pregnancy. 
Awareness of such a condition is needed to avoid delay in diagnosis and management. Detailed imaging of the abdomen, including the upper abdomen should be done whenever required. Some authors have also advised for computerized tomography (CT scan). ${ }^{7}$ Role of laparoscopy cannot be understated, as it ensures the inspection of the entire abdominal cavity without the need for a large abdominal incision. In suspected ectopic pregnancy, the upper abdomen should be thoroughly inspected if the adnexa are found missing from the pelvic location. Renal anomalies like agenesis, pelvic kidney and horse-shoe shaped kidney, are common in patients with unicornuate uterus. In this case also, right sided renal agenesis was observed.

\section{CONCLUSION}

The case is reported due to its rarity and the diagnostic dilemma associated with it. Ectopic pregnancy can occur in an undescended non-communicating fallopian tube seen with unicornuate uterus with absent horn. Diagnosis should be suspected in patients with unilateral absent pelvic adnexa, if other features favour ectopic pregnancy. Patients with incidentally diagnosed unicornuate uterus with non-communicating fallopian tube should undergo salpingectomy to prevent future ectopic pregnancy.

Funding: No funding sources Conflict of interest: None declared

Ethical approval: Not required

\section{REFERENCES}

1. Taran FA, Kagan KO, Hübner M, Hoopmann M, Wallwiener D, Brucker S. The diagnosis and treatment of ectopic pregnancy. Deutsches Ärzteblatt International. 2015;112(41):693.

2. Grimbizis GF, Gordts S, Di Spiezio Sardo A, Brucker S, De Angelis C, Gergolet M, et al. The ESHRE/ESGE consensus on the classification of female genital tract congenital anomalies. Hum Reprod. 2013;28:2032-44.

3. Helvacioglu A, Gilmore S, Willson W, Rizk B. Ovarian malposition-mullerian anomalies revisited. Middle East Fertil Society J. 2010;15(2):115-8.

4. Pardal C, Braga L, Vides B. Ectopic pregnancy in an undescended fallopian tube: a diagnostic challenge. BMJ Case Reports. 2016;2016:bcr2015213058.

5. Gnanasambanthan S, Uchil D. A rare case of an ectopic pregnancy in the rudimentary tube of a patient with an isolated unicornuate uterus. BMJ Case Reports. 2017;2017:bcr-2017.

6. Seoud MA, Khayyat H, Mufarrij IK. Ectopic pregnancy in an undescended fallopian tube: an unusual presentation. Obstetr Gynecol. 1987;69:4557

7. Woolnough B, Ballermann C. An atypical presentation of ectopic pregnancy with unicornuate uterus and undescended fallopian tube. J Obstet Gynaecol Canada. 2019;41(2):214-6.

8. Nielsen KG, Pedersen AT. An ectopic pregnancy under the liver. Case Reports. 2010;2010:bcr0120102615.

Cite this article as: Brahmbhatt $S$, Makhija A, Brahmbhatt J, Patel YN. Ectopic pregnancy in a case of congenital mullerian anomaly: a diagnostic dilemma. Int J Reprod Contracept Obstet Gynecol 2020;9:3512-4. 